\title{
Implicit and Explicit Stereotyping of Adolescents
}

\author{
Elisheva F. Gross ${ }^{1}$ and Curtis D. Hardin ${ }^{2}$
}

\begin{abstract}
Although adolescents are commonly assumed to be rebellious, risky and moody, two experiments demonstrate for the first time that these beliefs operate both explicitly and implicitly as stereotypes. In Experiment 1, participants (a) explicitly endorsed adolescent stereotypes and (b) implicitly associated adolescent stereotyped words more rapidly with the adolescent than the adult social category. Individual differences in the explicit endorsement of adolescent stereotypes predicted explicit perceptions of the rebelliousness of a 17-year-old but not a 71-year-old, although individual differences in implicit stereotyping did not. Identification with adults was associated with greater implicit stereotyping but not explicit stereotyping. In Experiment 2, subliminal exposure to adolescent stereotyped words increased subsequent perceptions of the rebelliousness of a 17-year-old but not a 71-year-old. Although individual differences in implicit adolescent stereotyping did not predict explicit evaluations of adolescents, stereotypes of adolescents nevertheless influenced explicit evaluations unconsciously and unintentionally.
\end{abstract}

KEY WORDS: implicit attitudes; prejudice; stereotyping; adolescents; intergroup conflict.

Adolescence has been noted for its threat to adult tranquility since Epicurus, and has been identified as a period of "storm and stress" by no less an authority than a founder of the American Psychological Association (Hall, 1904). Yet only recently has the common characterization of adolescents as rebellious, risky, and moody been identified as a stereotype,

\footnotetext{
${ }^{1}$ University of California, Los Angeles, CA, USA.

${ }^{2}$ Department of Psychology, Brooklyn College \& Graduate Center, City University of New York, Brooklyn, NY 11210-2889, USA.

${ }^{3}$ Address correspondence to: Curtis D. Hardin, Department of Psychology, Brooklyn College \& Graduate Center, City University of New York, Brooklyn, NY 11210-2889, USA., e-mail: cdhardin@brooklyn.cuny.edu
} 
endorsed by college students, teachers, and parents alike (Buchanan et al., 1990; Buchanan and Holmbeck, 1998; Graham and Lowery, 2004; Holmbeck and Hill, 1988). Although the ubiquity of beliefs about adolescents might suggest that adolescents are very likely to be stereotyped, there is to date no experimental evidence that these beliefs actually operate as stereotypes in social judgment-i.e., guiding perceptions over and above behavior.

If explicit, conscious beliefs about adolescents operate as stereotypes, then the stereotypes also may operate "implicitly." Indeed, other common stereotypes involving ethnicity and gender are known to operate in the absence of the perceiver's intentions, conscious awareness, or control (for reviews see Greenwald and Banaji, 1995; Hofmann et al., 2005). In research on stereotyping and prejudice of African-Americans, women, and the elderly, for example, explicit and implicit attitudes may even operate with apparent independence (e.g., Blair and Banaji, 1996; Greenwald et al., 2002; Henry and Hardin, 2006; Perdue and Gurtman, 1990). For example, the degree to which participants unintentionally associate traditionally gendered vocations like nursing with women and construction with men is unmoderated by participant gender or participant willingness to explicitly endorse gender stereotypes (Banaji and Hardin, 1996).

There are several rigorous ways to test if stereotypes are being used in social judgment, and we have integrated the most important of these to demonstrate that common beliefs about adolescents are indeed used as stereotypes in judgments of adolescents. One test is to assess the degree to which individual differences in endorsing a stereotype discriminately predict perceptions of stereotyped social targets. For example, is a given adolescent (but not an adult) perceived as more rebellious to the extent that one endorses stereotypes of adolescents? A second test is to assess the degree to which common beliefs about a group are more easily-or even automatically - associated with that group compared to other groups. For example, are words like "moody" and "risky" and "rebellious" more easily associated with adolescents than adults? A third test is to assess the degree to which an experimental manipulation of the cognitive accessibility of a stereotype discriminately affects perceptions of stereotyped social targets. For example, is a given adolescent (but not an adult) perceived as more rebellious when adolescent stereotypes are cognitively salient?

The third test integrates two signature indicators of stereotype use in social cognition research, one in which stereotype use is revealed by effects of the relative accessibility of the stereotype on perceptions of a given social target and another in which stereotype use is revealed by effects of the relative applicability or relevance of a stereotype to a given social 
target. For example, a stereotype-accessibility effect is illustrated by the finding that a race-unspecified target was judged to be especially hostile among participants who had recently been exposed to words associated with common American stereotypes of African Americans (Devine, 1989). A stereotype-applicability effect is illustrated by the finding that thoughts about dependence increased perceptions of the dependence of a female target but not a male target, whereas thoughts about aggression increased perceptions of the aggressiveness of a male target but not a female target (Banaji et al., 1993). Although social cognition research on stereotyping has long been dominated by stereotype-accessibility effects and theorizing (e.g., Devine, 1989), applicability is as important as accessibility in determining the course of information use in social judgment, including stereotyping (e.g., Hardin and Rothman, 1997; Higgins, 1996; Higgins et al., 1977). In fact, effects of stereotype accessibility in the absence of applicability conditions are difficult to distinguish from run-of-the-mill effects of semantic relation.

\section{OVERVIEW OF THE PRESENT RESEARCH}

In this research we tested for the first time whether adolescent stereotypes are discriminately used in perceptions of adolescents. In Experiment 1, we assessed the degree to which perceptions of a 17-yearold (but not a 71-year-old) were predicted by both individual differences in (a) the explicit endorsement of adolescent stereotypes, and (b) the implicit stereotype-related associations people have with adolescents. In Experiment 2, we manipulated the cognitive accessibility of adolescent stereotypes and observed the effect on subsequent perceptions of a 17-year-old and a 71-year-old. In both experiments, the stereotypical trait evaluated by participants was rebelliousness. Rebelliousness is the most common association with adolescents and the most strongly endorsed characteristic of adolescents in both our own pilot research and in previous research (e.g., Buchanan and Holmbeck, 1998; Holmbeck and Hill, 1988). The strategy of focusing on a single, highly stereotypical trait has proved useful in investigations of other common stereotypes, including those related to race (e.g., Devine, 1989) and gender (e.g., Banaji et al., 1993). In both of our experiments, participants evaluated a social target on the basis of a paragraph describing a series of behaviors plausibly related to the stereotype of interest (e.g., Banaji et al., 1993; Devine, 1989; Higgins et al., 1977). The procedure allows an investigation of stereotype use independent of the target's behavior as well as the discriminate application of the stereotype to a stereotyped versus non-stereotyped target. 


\section{EXPERIMENT 1}

This experiment tested the proposition that common stereotypes of adolescents are implicitly and explicitly used in judgments of adolescents, and applied discriminately. In an initial session participants evaluated either a teenaged or elderly person on traits both related and unrelated to a common adolescent stereotype. In two subsequent, ostensibly unrelated sessions conducted several weeks after the first session, participants completed (a) a measure of explicit endorsement of adolescent stereotypes, (b) measures of contact and identification with adolescents and adults, and (c) a measure of implicit stereotyped associations with adolescents. Stereotyping was examined by assessing the degree to which explicit and implicit stereotyping discriminately predicted rebellious-related versus rebellious-unrelated judgments of the adolescent target versus the elderly target.

\section{METHOD}

\section{Participants}

Students enrolled in a social psychology course at U.C.L.A. (84 female, 22 male) participated for course credit. The sample was $44 \%$ European American, 36\% Asian American, 16\% Latino, 3\% African American, and $1 \%$ Native American. Twenty-two percent of the participants were born outside the United States and had lived in the U.S. an average of 13.6 years $(\mathrm{SD}=8.0)$. The average participant age was 21.5 years old $(\mathrm{SD}=4.2)$.

\section{Materials}

\section{Social Judgment Task}

Participants were told that the experiment concerned how people form impressions of others. They read and evaluated a 12-sentence paragraph describing an incident in which an individual, "D.," is stopped by a police officer for disregarding a stop sign (Appendix A). D. was identified in the first sentence as either 17 years old or 71 years old; the rest of the paragraph was identical in both conditions. Pilot data suggested that the nature of the traffic violation and D.'s behavior towards the officer were considered to be sufficiently plausible and ambiguously rebellious for both a 17- and 71-yearold. 
After reading the paragraph, participants rated D. on each of 8 traits on scales that ranged from 1 (not at all) to 7 (extremely). In the tradition of previous research on stereotyping (e.g., Banaji et al., 1993; Devine, 1989), we examined the degree of stereotype specificity by including an equal number of both positive and negative traits that are related versus unrelated to the focal trait of rebelliousness (as judged by participants in Anderson, 1968 and our own pilot studies). The scales thus included 2 positive rebellious-related words (adventurous, daring), 2 negative rebellious-related words (rebellious, disobedient), 2 positive rebellious-unrelated words (relaxed, shy) and 2 negative rebellious-unrelated words (vulgar, squeamish).

\section{Explicit Stereotyping}

To assess explicit adolescent stereotyping, the nine-item "Storm and Stress Beliefs Scale" was used (Holmbeck and Hill, 1988). The scale has been validated on both adult and college student populations. On seven-point scales $(1=$ never true to $7=$ always or almost always true $)$, participants indicated the degree to which they believe that particular characteristics are true of adolescents. Examples include: "Adolescence is a stormy and stressful time," and "Adolescents frequently fight with their parents."

\section{Implicit Stereotyping}

Implicit stereotyping of adolescents was assessed by the Implicit Association Task (IAT; e.g., Greenwald et al., 1998; Lowery et al., 2001) in which implicit stereotyping is indicated by the degree to which people unintentionally associate beliefs about a group to representative exemplars of the group (cf. Greenwald and Banaji, 1995). ${ }^{1}$ The IAT is an interference paradigm, like the classic Stroop (1935) procedure, in which associational strength is inferred by the degree to which one categorization task creates behavioral interference in another concurrently performed categorization task. To assess stereotyped associations about adolescents, participants simultaneously made two categorical judgments as quickly and accurately as possible - judging one set of words (e.g., teenager, youth, grown-up, lady) as "adolescent" versus "adult," and judging another set of words (e.g., stubborn, rebellious, table, chair) as "adjective" versus "furniture." Adolescent

\footnotetext{
${ }^{1}$ Although the IAT is nominally "implicit," it arguably may better be characterized as "automatic," in the sense that, regardless of one's awareness that it is a measure of stereotyping or prejudice, associational strength does not appear vulnerable to conscious intentions to control task performance (cf. Blair and Banaji, 1996; Blair et al., 2001; Lowery et al., 2001)
} 
stereotyped adjectives were selected from (a) descriptors provided by 30 pilot test volunteers at the Los Angeles airport who listed their own and others' associations with adolescents or teenagers, and (b) items included in the Beliefs About Adolescent Personality and Behavior scale (Buchanan and Holmbeck, 1998). The category "furniture" was selected as a standard of comparison because participants in our sample do not differentially associate furniture with adolescents and adults, thereby making it an estimate of the degree to which putative characteristics of adolescents (e.g., rebelliousness) are associated with adolescents versus adults, independently of stereotypes of adults. Implicit stereotyping is indicated by the degree to which stereotype-congruent judgments are faster than stereotype-incongruent judgments.

In each critical phase of the IAT, participants were presented with 40 words from all four categories (teenager labels, adult labels, stereotypic adjectives, and furniture words) in a list down the center of a page. Association strength was estimated by the number of judgments made in $20 \mathrm{~s}$ (e.g., Lowery et al., 2001). Adolescent stereotyping was estimated by the degree to which more correct judgments were completed in the stereotype congruent phase than the stereotype incongruent phase - that is, the degree to which participants completed more judgments when their behavior associated adolescent stereotypes with adolescents than adults. Appendix B shows a stimulus sheet for the stereotype-incongruent phase of judgments. Practice blocks of judgments preceded each of two critical (congruent and incongruent) phases. The order of both the phases and the words in each list were counterbalanced across all participants. Neither factor qualified the reported results.

\section{Identification, Contact, and Affect Measures}

To explore the possibility that adolescent stereotyping may be related to social identification processes implied by several theories, including selfcategorization theory (e.g., Turner, 1987), shared reality theory (Hardin and Conley, 2001; Hardin and Higgins, 1996), and balance theory (Heider, 1958), we assessed participant perceptions of their social contact and identification with adolescents and adults using eight items. Across six questions, participants indicated the degree to which they, their friends, and their family identify them as adult or adolescent (e.g., "For the most part, how much do your friends consider you to be an adolescent?" Responses were made on a scale ranging from 1 (not at all) to 7 (very much). Friendly contact was measured as the percentage of (a) teenaged and (b) adult friends each participant reported having. Additionally, participants indicated their affect towards both adolescents and adults on a standard thermometer scale ranging from 1 (very cool) to 100 (very warm). 


\section{Procedure}

Participants individually completed the social judgment task in groups of 3 to 10 for course credit. Five to seven weeks later, groups of 8 to 10 participants completed the explicit stereotyping measure and the contact and identification measures as part of an ostensibly unrelated experimental session, for which they also received course credit. The implicit stereotyping task was conducted as part of a third session - six to eight weeks after the social judgment task and at least a week after the second session-in a laboratory demonstration.

\section{RESULTS AND DISCUSSION}

Results suggest that not only are stereotypes of adolescents explicitly endorsed - and implicitly associated with adolescents - but that explicit (but not implicit) endorsement of adolescent stereotypes predicts social judgment of adolescent (but not elderly) social targets. Implicit stereotyping was predicted by the degree to which participants identified with adults and reported friendly contact with adults.

\section{Explicit Stereotyping}

Results demonstrated that the adolescent stereotype was explicitly endorsed and that it predicted social judgment. The explicit measure of adolescent stereotyping was normally distributed, exhibited acceptable reliability (Cronbach's alpha $=.73$ ), and revealed that participants endorsed adolescent stereotypes, as indicated by a mean score of $4.66(S D=0.6)$. In addition, participants perceived the 17-year-old target as more rebellious to the degree that they explicitly endorsed stereotypes about adolescents more than a month later, $r(71)=.34, p=.004$. The association was highly specific. Explicit stereotyping was related neither to ratings of the adolescent target on traits semantically unrelated to rebelliousness ratings $(r(71)=.12$, $p>.3$ ) nor to ratings of the 71-year-old target, whether related to rebelliousness $(r(71)=-.04, p>.5)$ or unrelated to rebelliousness $(r(71)=.06$, $p>.5)$. As shown in Table I no other significant associations with explicit stereotyping were observed.

\section{Implicit Stereotyping}

Implicit stereotyping was demonstrated by the finding that adolescent stereotyped words were more easily associated with words representing the 


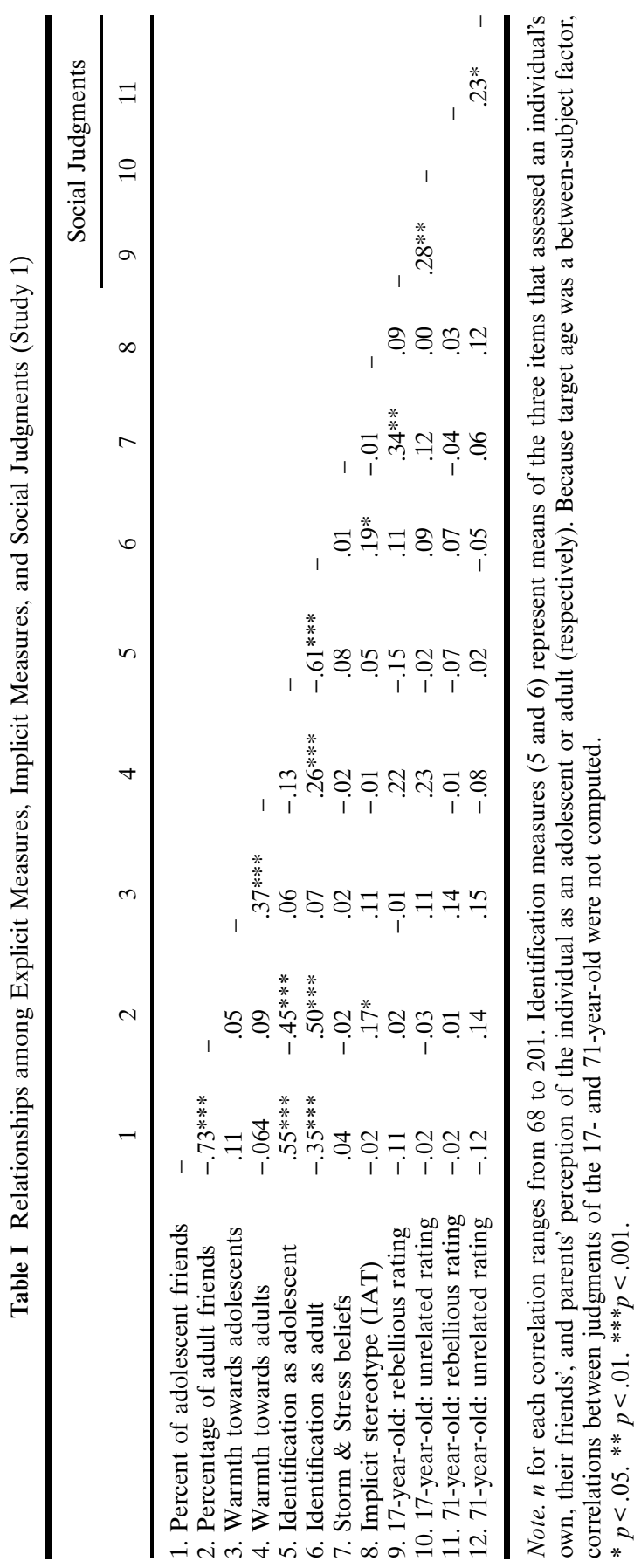


adolescent social category than the adult social category, and moreover that the strength of this association was predicted by the degree to which participants identified with adults and reported friendly contact with adults. Evidence that the stereotypes assessed in explicit measures are unintentionally associated with adolescents was indicated by performance on the IAT, which was normally distributed and revealed a mean stereotyping bias. On average, participants were able to correctly classify more words in the stereotype-congruent phases $(M=20.95, \mathrm{SD}=5.7)$ than in the stereotype-incongruent phases $(M=16.34, S D=4.9)$; the difference from zero $(M=4.61, S D=5.5)$ was statistically significant, $t$ $(205)=12.02, p<.001$, $e^{2} a^{2}=.15$. Evidence that implicit stereotyping of adolescents was common in this sample was indicated by the finding that $75 \%$ of the participants $(n=154)$ showed the stereotyping bias, whereas 8 $\%(n=17)$ showed no bias, and $17 \%(n=35)$ showed a counter-stereotypical bias. Implicit stereotyping did not differ reliably by participant age, ethnicity, or gender.

Replicating a common dissociation between implicit and explicit attitudes in previous research (Blair, 2001), the implicit and explicit stereotyping measures were uncorrelated, $r(132)=-.01, p>.9$. Unlike explicit stereotyping, implicit stereotyping was associated with adult contact and identification. Implicit adolescent stereotyping was greater to the degree that participants identified adults as friends, $r(132)=.17, p<.05$, and to the degree that participants identified themselves as adults, $r(132)=.19$, $p<.05$, similar to some relations between interpersonal contact and implicit attitudes that have been recently identified (cf. Henry and Hardin, 2006; Rudman et al., 2001). However, no significant associations were detected between implicit stereotyping and any of the explicit social evaluations across target and trait type.

Given that the IAT scores were predictive of neither explicit stereotypical beliefs nor judgments of the adolescent target, one might reasonably ask how the existence of implicit adolescent stereotyping is affirmed at all? One answer is that the adolescent-stereotype association captured in this experiment is itself prima facie evidence of implicit stereotyping - it is, literally, a tendency to discriminately associate stereotypically adolescent characteristics with words representing the adolescent social category. Nevertheless, further research is necessary to identify the conditions, if any, in which individual differences in implicit adolescent stereotyping predict other attitudes and behaviors about adolescents (Blanton and Jaccard, 2006; Pelham et al., 2005).

Although individual differences in implicit adolescent stereotyping were not associated with more stereotyped perceptions of the adolescent target in Experiment 1, this does not preclude the possibility that adolescent stereotypes nevertheless may implicitly influence perceptions of adolescents. In 
fact, the social cognition literature is replete with demonstrations of the unconscious, unintentional use of stereotypes in social judgment in the absence of effects of individual differences in implicit or explicit stereotyping. In this literature, implicit stereotyping is indicated when social perceptions are more stereotype-consistent when the stereotype is relatively more salient at the time of judgment. Notably, one of the most striking things about Devine's (1989) classic demonstration of implicit racial stereotyping was the fact that it occurred to an equal degree whether participants were high or low in explicit racial prejudice.

\section{EXPERIMENT 2}

In Experiment 2 we adopted a common social-cognition paradigm to test the proposition that judgments of adolescents are implicitly and discriminately affected by the cognitive accessibility of adolescent stereotypes (e.g., Banaji et al., 1993; Devine, 1989; Higgins et al., 1977). Two critical components - "accessibility" and "applicability"—are necessary to demonstrate implicit stereotyping in this paradigm. Cognitive accessibility is a kind of top-of-the-head phenomenon characterized by an easy readiness to use information in judgment, and is a foundational construct in social-cognition theorizing about stereotyping (e.g., Devine, 1989; Higgins, 1996). The accessibility component is captured by the question: Is social judgment more stereotyped when the stereotype is mentally salient than when it is not salient? The second component is applicability, which is the relevance of accessible information to the judgment at hand. Highly accessible information may or may not be used in a given social judgment, and is not used if it is inapplicable (e.g., Higgins, 1996). The applicability component is characterized by the question: Does stereotype salience discriminately affect judgment of a stereotyped versus non-stereotyped target? Although applicability is rarely manipulated in stereotyping experiments (but see Banaji et al., 1993; Higgins et al., 1977), implicit stereotyping effects are always predicated on it (Hardin and Rothman, 1997). We have chosen to integrate the common accessibility approach with the less common applicability approach as a means to triangulate on the discriminate operation of adolescent stereotypes in judgments of adolescents on a stereotype-relevant dimension of judgment.

In this experiment, participants evaluated either a 17 -year-old or a 71year-old who behaved in an identically rebellious manner after subliminal exposure to words either related or unrelated to the adolescent stereotype. We hypothesized that exposure to the adolescent stereotype would increase 
rebellious (but not rebellious-unrelated) judgments of the adolescent target (but not the elderly target).

\section{METHOD}

\section{Participants}

Thirty-seven UCLA introductory psychology students (19 female, 18 male) participated for course credit. The sample was 43\% European American, 41\% Asian American, 11\% Latino, and 5\% African American. Thirty percent of participants were born outside of the United States and had lived in the U.S. an average of 9.5 years $(\mathrm{SD}=5.3)$. The average participant age was 20.8 years old $(\mathrm{SD}=2.5)$.

\section{Materials}

The social judgment task was identical to the one used in Experiment 1. The stereotype salience manipulation was a variation of a widely employed paradigm in which participants are subliminally exposed to words on a computer screen while they complete a perceptual reaction time task ostensibly unrelated to the subsequent social judgment task. The procedure, adapted from Devine (1989), was administered using PsyScope software (Cohen et al., 1993) on Macintosh computers with screen refresh cycles of $17 \mathrm{~ms}$. Participants focused on a fixation point marked " $\mathrm{xx}$ " in the center of the screen while judging the location of a series of random letter strings presented parafoveally in one of four quadrants of the screen. Participants indicated as quickly and accurately as possible the location of each letter string by pressing an appropriately labeled key: " $\mathrm{L}$ " for left, " $\mathrm{R}$ " for right. Distinctive beeps followed each key response to indicate correct or incorrect judgments. For each of 5 practice and 88 trials, participants were exposed to a prime stimulus word for $68 \mathrm{~ms}$ ( 4 screen refresh cycles) immediately followed by a masking stimulus, a random letter string, for $85 \mathrm{~ms}$, and an intertrial interval of $200 \mathrm{~ms}$. Pre-testing confirmed that this procedure was effective in presenting the priming words outside of participants' awareness. After each of 88 trials, none of ten pre-test participants asked to indicate whether they had seen a word (and if so, what word) correctly identified a single word presented on any of the trials. 
Participants were randomly assigned to exposure to words judged by pre-test volunteers to be either highly related or unrelated to stereotypes of adolescents $^{2}$. Stereotypic and non-stereotypic words were equal on average word length (7.6 letters). In addition, the non-stereotypic words were selected to be equally or more negative than the stereotypic words, as judged by either college student participants in previous research (Anderson, 1968) or our own pre-testing.

\section{Procedure}

After completing a demographic questionnaire, participants completed the stereotype priming task, followed by the social judgment task, individually or in small groups. A manipulation check conducted in writing and then verbally during debriefing probed participants for (a) the age of the social judgment target, D., (b) what words, if any, they saw flash on the screen during the perceptual task, and (c) their perceptions and/or suspicions concerning the experiment's aims and hypotheses. No participant correctly identified a single word to which they had been subliminally exposed or indicated any suspicion that the priming task affected their judgment in any way.

\section{RESULTS AND DISCUSSION}

Results indicated that not only does subliminal exposure to adolescent stereotyped words affect social judgment, but that the effect is specific to the adolescent target. As shown in Fig. 1, participants judged the 17-year-old but not the 71-year-old as more rebellious after subliminal exposure to adolescent stereotype-related words than unrelated words. Although the default ANOVA Prime x Target x Judgment Dimension 3-way interaction was marginally significant, $F(1,37)=3.63, p=.07$, et $a^{2}=.10$, the test does not accurately model the predicted pattern of results (see Abelson, 1995; Rosenthal and Rosnow, 1985). The specific interaction we predicted was obtained, as indicated by a significant planned contrast that assigned coefficients of -1 and 1 to the rebelliousness judgments of the 17-year-old target

${ }^{2}$ Each of the following words appeared four times in the stereotype condition: acne, adolescent, anxious, awkward, depressed, emotional, hormones, hot-tempered, identity, immature, impulsive, insecure, moody, puberty, rebellious, rude, self-conscious, selfish, stubborn, teen, teenager, young, youth. Each of the following words appeared four times in the non-stereotype condition: hatred, combination, tired, meek, scolding, unlucky, termite, finicky, moment, dominating, shy, unentertaining, inefficient, factory, spiteful, undecided, vulgar, unimaginative, engine, ship, practical, diamond, stern. 

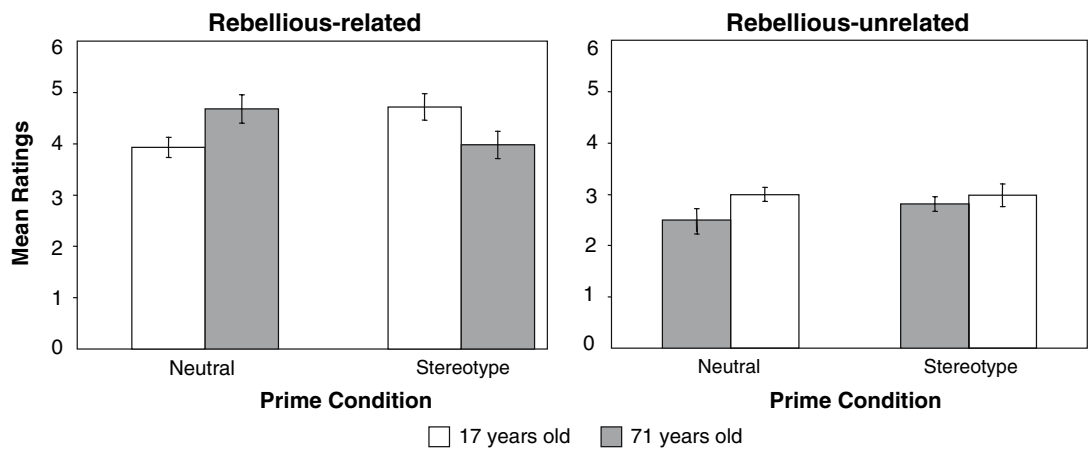

Fig. 1 . Average ratings of rebellious-related and unrelated traits by age of target and prime condition.

in stereotype and neutral prime conditions, respectively, and coefficients of 0 to all others, $F(1,37)=5.33, p=.027$, eta $^{2}=.14$. The finding was explored further by 2-way Target Age×Stereotype Salience ANOVAs for stereotyperelated and unrelated judgments separately. Although the adolescent priming manipulation affected judgments of the rebellious-related traits, $F$ (1, $37)=8.82, p=.006$, eta ${ }^{2}=.21$, no such effect was detected on the rebellious-unrelated judgments, $F(1,37)=1.05, p=.31$, eta ${ }^{2}=.03$. The 17-yearold was judged significantly more rebellious after subliminal exposure to adolescent stereotype-related words than stereotype-unrelated words, $t$ $(18)=-2.47, p<.03, e t a^{2}=.25$, but the 71-year-old was judged marginally less rebellious after subliminal exposure to adolescent stereotype-related words than unrelated words, $t(15)=1.78, p=.10$, eta ${ }^{2}=.17$. Judgments of the 71-year-old target suggest that the stereotype may have provided a standard against which participants contrasted the older target (see Banaji et al., 1993; Biernat and Manis, 1994), resulting in judgments of the elderly target as marginally less rebellious after exposure to adolescent stereotyped words than non-stereotyped words. This possibility is congruent with the finding that the 71-year-old was judged to be more rebellious than the 17year-old among participants exposed to non-stereotypical words, $t$ $(17)=-2.24, p=.038$, eta $^{2}=.23$. Though the target behavior described in the target paragraph was identical, results suggest that the behavior seemed somewhat more rebellious for an elderly person than an adolescent unless the adolescent stereotype had been made salient.

Although women judged the adolescent as more rebellious than did men, a significant Participant Gender $\times$ Target Age interaction, $F$ (1, $37)=4.62, p<.05$, eta $^{2}=.14$ (cf. Holmbeck and Hill, 1988), women and men were equivalently affected by the stereotype priming manipulation on rebelliousness judgments, as indicated by a nonsignificant Participant 
Gender $\times$ Stereotype Prime $\mathrm{x}$ Target Age $\times$ interaction, $F(1,37)=1.87$, $p=.18$, $e t a^{2}=.06$. In addition, the pattern of results did not differ by age, or degree of explicit endorsement of a "Storm and Stress" stereotype, replicating previous research showing no relation between individual differences in explicit stereotyping and the degree to which temporarily salient stereotypes are used in social judgment (e.g., Banaji et al., 1993; Devine, 1989).

Amid results that fit our predictions, interesting complications were also observed. Recall that four traits, two positive and two negative, comprised each type of judgment (rebellious-related or unrelated). Although the composite ratings operated as expected and reported, the individual traits did not conform equally. MANOVAs conducted on the four items in each rating index indicated that the stereotype exposure effects were highly specific. Among rebellious-related traits, only negative words (rebellious, disobedient) were subject to the Target agexExposure condition effect (rebellious: $F(1,37)=8.74, p=.006$, eta ${ }^{2}=.22$; disobedient: $F(1$, $37)=7.29, p=.01$, eta $\left.{ }^{2}=.19\right)$. The positive terms denotatively related to rebelliousness, adventurous and daring, yielded null effects $(p s=.13$ and .37 , etas $^{2}=.07$ and .03 , respectively). Moreover, the experimental manipulation significantly affected one of the negative terms in the rebellious-unrelated index, vulgar, $F(1,37)=4.84, p=.036$, eta ${ }^{2}=.14$.

Although interesting, it is important to note that the selective effect of adolescent stereotype activation on judgments of negative, confrontational traits cannot be dismissed as a mood or valence effect for several reasons. First, the non-stereotyped priming stimuli were chosen to be at least as negative as the stereotype priming stimuli. Most importantly, the effect of the priming manipulation only affected judgments of adolescents. The selective effect of the prime on evaluations of negative but not positive rebellious-related traits may, however, be at least partially a function of the target paragraph we employed. D.'s behaviors may have been more ambiguously disobedient and rebellious than ambiguously adventurous or daring, thereby making the positive, rebelliousness related traits inapplicable to the judgment at hand (see Hardin and Rothman, 1997; Higgins, 1996; Higgins et al., 1977). For example, the paragraph used by Higgins et al., (1977) was created to be equivalently applicable to the traits "reckless" and "adventurous." An explanation for the effect of the stereotype on ratings of adolescent (but not elderly) vulgarity awaits further investigation. Perhaps in the context of the stereotype and the specific scenario we employed the word vulgar may have appeared more related to rebelliousness than it appeared to pre-test participants who evaluated the traits in the absence of the adolescent stereotyping context.

In summary, the central finding of Study 2 may be restated in more specific terms: Compared to participants primed with words that were 
neutral with respect to the adolescent stereotype, participants subliminally exposed to stereotype-related words judged a 17-year-old but not a 71-yearold to be more rebellious, disobedient, and vulgar, but not more adventurous or daring. It thus appears that, at least under the conditions of this experiment, adolescents who behave in an ambiguously rebellious manner are subject to stereotypically negative evaluations and do not receive the benefit of the semantically related positive characteristics. This finding is also consistent with Buchanan and Holmbeck's (1998) finding that adolescents are stereotyped as more rebellious and risk-taking, but less adventurous than elementary school students.

\section{GENERAL DISCUSSION}

Age, race and gender are important dimensions of person categorization because they are visually perceived, rapidly triggered, and culturally meaningful (e.g., Beckett and Park, 1995). Although each of the "Big Three" categories has received considerable attention in social cognitive research on stereotyping (Fiske, 1998), until now research on age-based biases, or "ageism," has been concerned exclusively with attitudes towards the elderly (Fiske, 1998; Nelson, 2002; Zebrowitz and Montepare, 2000).

Addressing this omission, we found converging evidence across two experiments for both the implicit and explicit use of adolescent stereotypes in social judgment. Explicit adolescent stereotyping was indicated by the finding that individual differences in the endorsement of adolescent stereotypes predicted the degree to which a 17-year-old (but not a 71-year-old) was judged as rebellious. Implicit adolescent stereotyping was indicated by the findings that (a) words associated with the adolescent stereotype were more easily associated with adolescents than adults on an Implicit Association Task, and (b) subliminal exposure to words associated with the adolescent stereotype increased perceptions of the rebelliousness of a 17 -year-old but not a 71-year-old. Given that each distinct indication of implicit stereotyping we studied has unique strengths and weaknesses, it is notable that we found evidence for implicit stereotyping using both methods. In short, in addition to whatever kernel of truth beliefs about adolescents may have, this research demonstrates that these beliefs also construct perceptions of adolescents over and above their behavior, and can affect judgments of adolescents implicitly and unconsciously.

This research also confirms the highly discriminate nature of stereotyping. In Experiments 1 and 2, ratings of the 17-year-old, but not the 71-year-old, were positively associated with the degree of participants' adolescent stereotype endorsement and stereotype accessibility, respectively. It is worth noting that in the absence of such applicability effects, it is 
difficult to disentangle effects of stereotyping per se from effects of semantic priming. For example, the demonstration that words associated with adolescents increases perceptions of the rebelliousness of a 17-year-old alone is consistent with the possibility that words associated with adolescents affect judgments of any plausibly rebellious social target, adolescent or not. Though such effects are interesting and even important (e.g., Devine, 1989), they do not strictly show that beliefs are connected to specific social groups - a requirement at the heart of how stereotyping is usually defined. However, in our experiment stereotyping was not demonstrated by stereotype accessibility alone but also by the fact that the accessible stereotyped information was used specifically in judgments of the stereotyped social target. As such, this research adds to a small but growing empirical literature demonstrating that "applicability" is as important as "accessibility" in the cognition of social judgment (Banaji et al., 1993; Hardin and Rothman, 1997; Higgins et al., 1977).

We found in Experiment 1 that implicit and explicit adolescent stereotyping was uncorrelated. Implicit stereotyping (as measured by the IAT) and explicit stereotyping (as measured by the Storm \& Stress Scale) were not only unrelated to each other but also discriminately related to other measures. Although individual differences in explicit endorsement of adolescent stereotypes discriminately predicted perceptions of rebelliousness of an adolescent target, individual differences in implicit stereotyping as assessed by the IAT did not. Moreover, implicit but not explicit stereotyping of adolescents was predicted by friendly contact and identification with adults. As such, these findings join a growing number of studies demonstrating the discriminate predictive validity of implicit and explicit measures of social attitudes (e.g., Blair, 2001; Dovidio et al., 2002; Fazio et al., 1995; Greenwald and Banaji, 1995; Pelham et al., 2005; Rudman, 2004; Spalding and Hardin, 1999; Wittenbrink et al., 1997).

Although Experiment 2 demonstrates that the adolescent stereotype can be used implicitly in judgments of adolescents versus adults, it is worth emphasizing that this hardly demonstrates that negative adolescent stereotyping is inevitable in social perceptions of adolescents under all conditions. Indeed, from our perspective, it is likely that implicit stereotyping of adolescents is qualified by any number of social and situational characteristics. To give just one example from the social cognition literature, implicit use of a negative adolescent stereotype may well elicit more positive evaluations of some adolescent targets when the stereotype is used as a standard of comparison for a relatively positive behavior (e.g., Biernat and Manis, 1994).

In light of empirical evidence that adolescents are in fact more likely than younger and older people to exhibit behaviors consistent with the adolescent stereotype, one might question why people's endorsement of a 
more or less accurate view should be considered "stereotyping." Stereotypes are indeed sometimes rooted in more or less accurate perceptions of target group members (e.g., Allport, 1954; Jussim, 1991). The present research demonstrates, however, that people are not simply engaged in perception of adolescent behavior alone. Given identical information, participants used stereotypes about adolescents in their judgments of members of the adolescent age group. They literally went "beyond the information given" (Bruner, 1973).

In sum, although stereotypes of adolescents may to a degree reflect actual adolescent behavior, they also may shape perceptions outside of the awareness and conscious control of the perceiver. Developmental researchers have acknowledged the impact on adolescent behavior of beliefs about the nature of adolescence (e.g., Paikoff and Brooks-Gunn, 1991). The present research demonstrates one way in which such beliefs may exert an influence on judgments of adolescent behavior. If adolescent stereotypes function like other common stereotypes, such as those involving gender and race, they may also contribute to the manifestation of storm and stress behaviors through behavioral confirmation and stereotype threat processes (e.g., Klein and Snyder, 2003; Steele and Aronson, 1995). However, this intriguing possibility awaits future investigation. For now, we suggest that to the famed alliterative descriptors of adolescence as "stormy" and "stressful," we must add "stereotyped."

\section{APPENDIX A}

Please read the following paragraph very carefully and form an impression of the main character, D. After reading this selection, you will be asked to rate $\mathrm{D}$. on a number of attributes.

D., age 17[71], is driving in his neighborhood one afternoon. He's almost home when a police officer pulls him over. For a second, D. considers speeding away. If he could just have taken that last turn instead, he would have avoided the cop! But instead, D. slows down and pulls off to the side of the road. The police officer arrives at his door, requests his license, insurance and registration, and then asks D. if he knows why he was stopped. D. rolls his eyes and sighs. "Yes, officer," he says, "the stop sign, right?" The officer replies sternly, "You ran right through it, in fact. Did you even see me following you?" The officer peers at him through the car window, and D. wonders if the cop is smelling around for alcohol. "What, do you think I'm drunk or something?" says D., as he shuffles through his glove compartment for his registration. 


\section{APPENDIX B}

The defining feature of the IAT is to procedurally link two categorization tasks through a single behavioral decision - in our case, marking the right or the left column. Stereotype-congruent blocks are those in which the same behavior - say, marking the left column - indicates both adolescent adjectives (e.g., rebellious, stubborn) and the adolescent social category (e.g., teenager, juvenile). Stereotype-incongruent blocks are those in which the same behavior - say, marking the left column - indicates both adolescent adjectives (e.g., rebellious, stubborn) and the adult social category (e.g., grown-up, lady). Implicit adolescent stereotyping is calculated for each participant by subtracting the number of correct judgments completed in the ADULT-ADJECTIVE phase from the number of correct judgments completed in the TEENAGER-ADJECTIVE phase. Average reaction times can be calculated by dividing the number of correct judgments for a given phase by 20 (i.e., the number of seconds allowed for each phase).

Defiant

WOMAN

Bureau

GROWN-UP

self-centered

YOUTH

Shelf

MAN

Invulnerable

TEEN

linen closet

JUVENILE

coat-rack

LADY

Stubborn

TEENAGER

Chair

ADULT

Motivated

YOUNG ADULT

Dresser

JUVENILE

Table

LADY

thrill-seeking

YOUTH

Loveseat

GENTLEMAN

Desk

ADOLESCENT 


\begin{tabular}{ll}
\hline TEENAGER OR FURNITURE & \\
\hline & ADULT OR ADJECTIVES \\
\hline & Trendy \\
TEEN & Crazy \\
MAN & Futon \\
YOUNG ADULT \\
Smart \\
ADULT \\
Fast \\
GENTLEMAN \\
Confident \\
WOMAN \\
Rug
\end{tabular}

\section{REFERENCES}

Abelson, R. P. (1995). Statistics as Principled Argument, Mahwah, NJ: Lawrence Erlbaum.

Allport, G. W. (1954). The Nature of Prejudice, New York: Addison-Wesley.

Anderson, N. H. (1968). Likableness ratings of 555 personality-trait words. J. Pers. Soc. Psychol., 9, 272-279.

Banaji, M. R., and Hardin, C. D. (1996). Automatic stereotyping. Psychol. Sci., 7, 136-141.

Banaji, M. R., Hardin, C. D., and Rothman, A. J. (1993). Implicit stereotyping in person judgment. J. Pers. Soc. Psychol., 65, 272-281.

Beckett, N. E., and Park, B. (1995). Use of category versus individuating information: Making base rates salient. Pers. Soc. Psychol. Bull., 21, 21-31.

Biernat, M., and Manis, M. (1994). Shifting standards and stereotype-based judgments. J. Pers. Soc. Psychol., 66, 5-20.

Blair, I. V. (2001). Implicit stereotypes and prejudice. In Moskowitz Gordon, B. (ed.), Cognitive Social Psychology: The Princeton Symposium on the Legacy and Future of Social Cognition, Lawrence Erlbaum, Mahwah, NJ, pp. 359-374.

Blair, I. V., and Banaji, M. (1996). Automatic and controlled processes in stereotype priming. $J$. Pers. Soc. Psychol., 70, 1142-1163.

Blair, I. V., Ma, J. E., and Lenton, A. P. (2001). Imagining stereotypes away: The moderation of implicit stereotypes through mental imagery. J. Pers. Soc. Psychol., 81, 828-841.

Blanton, H., and Jaccard, J. (2006). Arbitrary metrics in psychology. Am. Psychol., 61, $27-41$.

Bruner, J. S. (1973). Beyond the Information Given, New York: Norton.

Buchanan, C. M., Eccles, J. S., Flanagan, C., Midgley, C., Feldlaufer, H., and Harold, R. D. (1990). Parents' and teachers' beliefs about adolescents: Effects of sex and experience. $J$. Youth. Adolesc., 19, 363-394.

Buchanan, C. M., and Holmbeck, G. N. (1998). Measuring beliefs about adolescent personality and behavior. J. Youth. Adolesc., 27, 607-627.

Cohen, J. D, MacWhinney B., Flatt M., and Provost J. (1993). PsyScope: An interactive graphic system for designing and controlling experiments in the psychology laboratory using Macintosh computers. Beh. Res. Methods, Instrum Comput. 25(2): 257-271.

Devine, P. (1989). Stereotypes and prejudice: Their automatic and controlled components. $J$. Pers. Soc. Psychol., 56, 680-690.

Dovidio, J. F., Kawakami, K., and Gaertner, S. L. (2002). Implicit and explicit prejudice and interracial interaction. J. Pers. Soc. Psychol., 82, 62-68.

Fazio, R. H., Jackson, J. R., Dunton, B. C., and Williams, C. J. (1995). Variability in automatic activation as an unobstrusive measure of racial attitudes: A bona fide pipeline?. J. Pers. Soc. Psychol., 69, 1013-1027. 
Fiske, S. T. (1998). Stereotyping, prejudice, and discripmination. In Gilbert, D. T. and Fiske, S. T. (eds.), The Handbook of Social Psychology, 2, McGraw-Hill, New York, NY, pp. 357411.

Graham, S., and Lowery, B. S. (2004). Priming unconscious racial stereotypes about adolescent offenders. Law. Hum. Behav., 28, 483-504.

Greenwald, A. G., and Banaji, M. R. (1995). Implicit social cognition: Attitudes, self-esteem, and stereotypes. Psychol. Rev., 102, 4-27.

Greenwald, A. G., Banaji, M. R., Farnham, S. A., Nosek, B. A., and Mellott, D. S. (2002). A unified theory of implicit attitudes, stereotypes, self-esteem, and self-concept. Psychol. Rev., $109,3-25$.

Greenwald, A. G., McGhee, D. E., and Schwartz, J. L. K. (1998). Measuring individual differences in implicit cognition: The Implicit Association Test. J. Pers. Soc. Psychol., 74, 1464-1480.

Hall, G. S. (1904). Adolescence: Its psychology and its Relation to Physiology, Anthropology, Sociology, Sex, Crime, Religion, and Education, Englewood Cliffs, NJ: PrenticeHall.

Hardin, C. D., and Conley, T. D. (2001). A relational approach to cognition: Shared experience and relationship affirmation in social cognition. In Moskowitz, G. B. (ed.), Future Directions in Social Cognition, Erlbaum, Hillsdale, NJ.

Hardin, C. D., and Higgins, E. T. (1996). Shared reality: How social verification makes the subjective objective. In Sorrentino, R. M. and Higgins, E. T. (eds.), Handbook of motivation and cognition: Foundations of social behavior Vol. 3, Guilford, New York, pp. 28-84.

Hardin, C. D., and Rothman, A. J. (1997). Rendering accessible information relevant: The applicability of everyday life. In Wyer, R. S. (ed.), Advances in Social Cognition, Erlbaum, Mahwah, NJ.

Heider, F. (1958). The Psychology of Interpersonal Relations, New York: John Wiley \& Sons.

Henry, P. J., and Hardin, C. D. (2006). The contact hypothesis revisited: Status bias in the reduction of implicit prejudice in the United States and Lebanon. Psychol. Sci., 17, 862868.

Higgins, E. T. (1996). Knowledge activation: Accessibility, applicability, and salience. In Higgins, E. T. and Kruglanski, A. (eds.), Handbook of Basic Principles, Guilford Press, New York.

Higgins, E. T., Rholes, W. S., and Jones, C. R. (1977). Category accessibility and impression formation. J. Exp. Soc. Psychol., 13, 141-154.

Hofmann, W., Gawronski, B., Gschwedner, T., Le, H., and Schmitt, M. (2005). A meta-analysis on the correlation between the implicit association test and explicit self-report measures. Pers. Soc. Psychol. Bull., 31, 1369-1385.

Holmbeck, G. N., and Hill, J. P. (1988). Storm and stress beliefs about adolescence: Prevalence, self-reported antecedents, and effects of an undergraduate course. J. Youth. Adolesc., 17, 285-306.

Jussim, L (1991). Social perception and social reality: A reflection-construction model. Psychol. Rev., 98, 54-73.

Klein, O., and Snyder, M. (2003). Stereotypes and behavioral confirmation: From interpersonal to intergroup perspectives. In Zanna, M. P. (ed.), Advances in Experimental Social Psychology Vol. 35, Academic Press, San Diego, pp. 153-234.

Lowery, B. S., Hardin, C. D., and Sinclair, S. (2001). Social influence effects on automatic racial prejudice. J. Pers. Soc. Psychol., 81, 842-855.

Nelson, T. D. (ed.) (2002). Ageism: Stereotypes and Prejudice against Older Persons, Cambridge, Mass: MIT Press.

Paikoff, R. L., and Brooks-Gunn, J. (1991). Do parent-child relationships change during puberty?. Psychol. Bull., 110, 47-66.

Pelham, B. W., Koole, S., Hardin, C. D., Hetts, J. J., and Seah, E. (2005). Implicit and explicit self-esteem and gender. J. Exp. Soc. Psychol., 41, 84-89.

Perdue, C. W., and Gurtman, M. B. (1990). Evidence for the automaticity of ageism. J. Exp. Soc. Psychol., 26, 199-216.

Rosenthtal, R., Rosnow, R. L. (1985). Contrast Analysis: Focused Comparisons in the Analysis of Variance, Cambridge University Press.

Rudman, L. A. (2004). Social justice in our minds, homes, and society: The nature, causes, and consequences of implicit bias. Soc. Justice Res., 17, 129-142. 
Rudman, L. A., Ashmore, R. D., and Gary, M. L. (2001). “Unlearning" automatic biases: The malleability of implicit prejudice and stereotypes. J. Pers. Soc. Psychol., 81, 856-868.

Spalding, L. R., and Hardin, C. D. (1999). Unconscious unease and self-handicapping: Behavioral consequences of individual differences in implicit and explicit self-esteem. Psychol. Sci., 10, 535-539.

Steele, C. M., and Aronson, J. (1995). Stereotype threat and the intellectual test performance of African Americans. J. Pers. Soc. Psychol., 69, 797-811.

Stroop, J. R. (1935). Studies of interference in serial verbal reactions. J. Exp. Psychol., 18, 643662.

Turner, J. C. (1987). Rediscovering the social group: Self-categorization theory, London: Blackwell.

Wittenbrink, B., Judd, C. M., and Park, B. (1997). Evidence for racial prejudice at the implicit level and its relationship with questionnaire measures. J. Pers. Soc. Psychol., 72, 262-274.

Zebrowitz, L. A., and Montepare, J. M. (2000). "Too young, too old": Stigmatizing adolescents and elders. In Heatherton, T. F. and Kleck, R. E. (eds.), The Social Psychology of Stigma, The Guilford Press, New York, pp. 334-373. 